\title{
Foreign Body Mimicking Sacral Tumour: Gauze Retained After the Lumbar Disc Surgery Performed 31 Years Ago
}

\section{Sedat Dalbayrak ${ }^{1}$, Salim Şentürk ${ }^{2 *}$, Onur Yaman ${ }^{2}$, Mesut Yılmaz $^{3}$ and Ali Fahir Özer ${ }^{2}$}

${ }^{1}$ Department of Neurosurgery, Neurospinal Academy, Istanbul, Turkey

${ }^{2}$ Deparment of Neurosurgery, Koç University, Istanbul, Turkey

${ }^{3}$ Department of Neurosurgery, Neurospinal Academy, Istanbul, Turkey

*Corresponding author: Salim Şentürk, Deparment of Neurosurgery, Koç University, Istanbul, Turkey, Tel: 0905058727579; E-mail: sedatdalbayrak@gmail.com Rec date: Apr 06, 2016; Acc date: May 28, 2016; Pub date: May 30, 2016

Copyright: ( 2016 Dalbayrak S, et al. This is an open-access article distributed under the terms of the Creative Commons Attribution License, which permits unrestricted use, distribution, and reproduction in any medium, provided the original author and source are credited.

\begin{abstract}
It is quite rare for foreign bodies retained after surgery to display tumour-like clinical and radiological findings. A 60 year-old female patient presented to the clinic with low-back and left hip pain. She had undergone lumbar disc surgery 31 years ago. She had pain in her low-back and left hip for 3 months. The patient's CT and MRI showed a well-defined mass lesion at left posterior S2-S3, measuring 3.5 to $4 \mathrm{~cm}$, with circumferential scattered contrast enhancement, which extended to the canal and caused bone erosion. Only when the mass was completely resected with its circumference, it was found out that it was the gauze retained after the surgical procedure. Our case is the oldest one in the neurosurgery and spinal surgery literature, which showed clinical findings 31 years after the lumbar disc surgery.
\end{abstract}

Keywords: Foreign body after surgery; Lumbar disc surgery; Sacral tumour

\section{Introduction}

Foreign bodies retained after surgical procedures manifest themselves in various clinical and radiological pictures. Infection, reactions and wound discharge associated with the foreign body are the most common clinical pictures [1]. Nevertheless, sometimes the foreign body may form giant atypical masses coupled with the reaction of the tissue surrounding it $[2,3]$. These atypical masses only rarely display tumour-like clinical and radiological findings.

This paper reports a case operated due a pre-diagnosis of sacral tumour, which later revealed "a sacral tumour mimicking foreign body" retained after the surgical procedure that had been performed 31 years ago.

\section{Case Report}

It was found out that the 60 -year-old female patient had undergone surgery 31 years ago due to low-back and left leg pain. It was found that the patient suffered from intermittent low-back and hip pain during the last 5 years, and her condition deteriorated during the previous year. Physical examination of the patient showed a scar tissue on the earlier surgical site. Laboratory tests did not reveal any pathological findings. Neurological examination showed left EHL $+4 / 5$. Her bladder and bowel functions were normal. Based on the tests, it was considered that she had undergone L5-S1 discectomy.

The patient's imaging studies showed a well-defined, heterogeneously dense mass lesion (Figures 1a, 1b, 2a and 2b) measuring 3.5 to $4 \mathrm{~cm}$ at left posterior S2-S3, with circumferential scattered contrast enhancement, which extended to the canal and caused bone erosion. Supplemental tests did not reveal any other pathology.

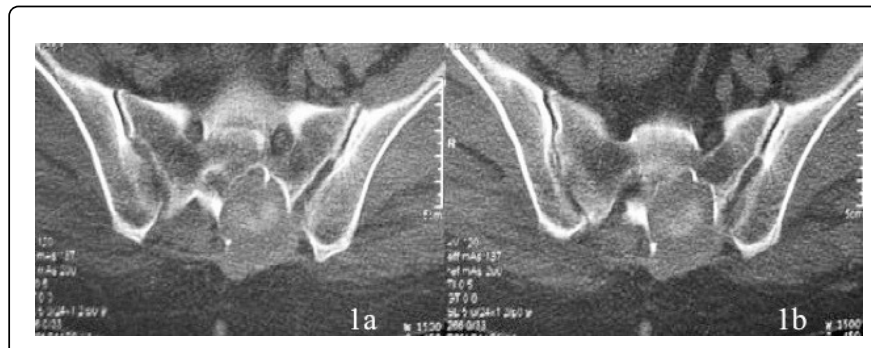

Figures 1a and 1b: Mass lesion at S2-S3, measuring 3 to $4 \mathrm{~cm}$ localizing on the left, which eroded posterior sacrum.

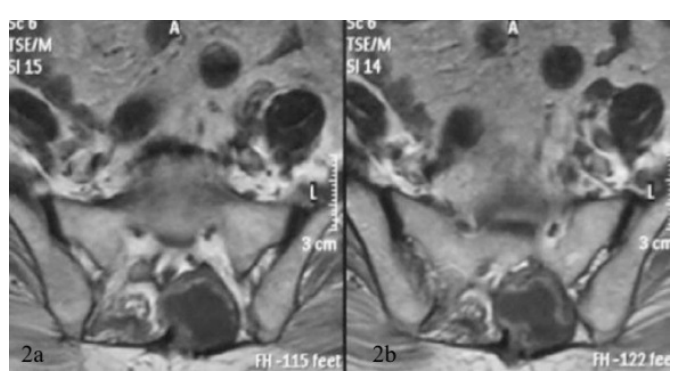

Figures 2a and 2b: MRI T1: Heterogeneous mass lesion at S2-S3 measuring 3 to $4 \mathrm{~cm}$ localizing at posterior sacrum under the fascia on the left.

The patient was operated based on the pre-diagnosis of sacral tumour, and the mass was resected en bloc with its circumference. A closer eye into the mass showed that it was the gauze retained after the lumbar disc surgery performed 31 years ago (Figures $3 a$ and $3 b$ ). 


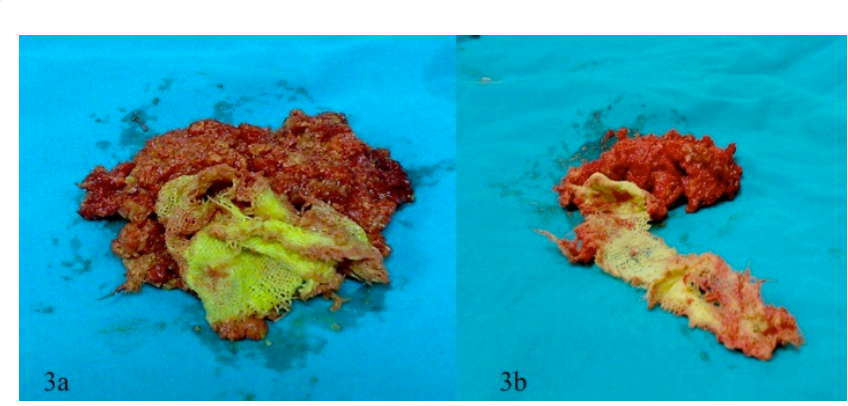

Figures $3 a$ and $3 b$ : Resected gauze.

\section{Discussion}

Gauze dressing, pads and cotton rolls are used as a routine in all surgery practices. These materials are predominantly used for hemostasis, dissection and retraction purposes especially in the spinal surgery practice since there is a lot of bleeding during surgery. Long and exhausting surgical procedures coupled with fatigue and carelessness result in these materials' being left at the surgical site during surgery. Figures are higher especially in obese patients [4]. The retained foreign body leads to various clinical and radiological findings mainly including infections, erosion of the surrounding tissue and compression [3]. They rarely show tumour-like clinical and radiological findings [2].

The retained foreign body usually manifests itself at early stages with persistent infection that does not respond to antibiotics therapy. Possible presence of a foreign body must be considered in case of recurrent post-operative infection and classical infection follow-up tests must be complemented with imaging studies to search for a foreign body. Reoperation decision should be taken based on the patient's imaging studies.

The retained foreign body usually induces two types of reactions: the first type is the aseptic fibrinous response surrounding the foreign body, resulting in granuloma formation. The second type is the exudative response leading to abscess formation. Secondary bacterial infections may or may not accompany some of the cases with this type of reaction [5]. These two types of reactions result in different MRI findings [2]. Our case presented with encapsulated foreign body granuloma.

Gossypiboma is underreported due to the professional and medicolegal concerns of surgeons. Likewise, the neurosurgery literature includes only a few papers on gossypiboma [6]. Ramirez and Thisted reported $0.7 \%$ gossypiboma after 10.000 lumbar disc surgeries [7]. A recent paper reported 6 cases of gossypiboma (0.24\%) after 2438 posterior lumbar spine surgeries [4].

The oldest case in the literature describes gauze retained after thoracotomy that had been performed 43 years ago. The oldest case in the neurosurgery literature is a patient who underwent lumbar disc herniation surgery 23 years ago. The patient who developed stenosis in the upper level showed a soft tissue tumour along the right paravertebral muscle planes at L5-S1 in the MRI. The encapsulated mass along the muscle planes was resected during the stenosis and disc surgery, and a closer eye revealed that it was the gauze [8]. However, this patient developed stenosis and disc related clinical findings, which were not associated with the gossypiboma. The oldest case with gossypiboma related clinical findings is a patient who underwent surgery 20 years ago due to T12 vertebral fracture [9]. Our case showed clinical findings after the longest time post-surgery in the neurosurgery and spinal surgery literature. No other diagnosis but sacral tumour was considered based on the tests.

We prefer en bloc resection of the lesion because lesion had regulary border.

\section{Conclusion}

Foreign body presence must be considered in the differential diagnosis of patients who were operated on the lesion site, even though the surgical procedure was performed many years ago.

\section{Conflict of Interest}

The authors declare that there are no potential conflicts of interest.

\section{References}

1. Mboti B, Gebhart M, Larsimont D, Abdelkafi K (2001) Textiloma of the thigh presenting as a sarcoma. Acta Orthop Belg 67: 513-518.

2. Karcnik TJ, Nazarian LN, Rao VM, Gibbons GE Jr (1997) Foreign body granuloma simulating solid neoplasm on MRI. Clin Imaging 21: 269-272.

3. Sahin-Akyar G, Yaqci C, Aytac S (1997) Pseudotumour due to surgical sponge: gossypiboma. Australas Radiol 41: 288-291.

4. Akhaddar A, Boulahroud O, Naama O, Al-Bouzidi A, Boucetta M (2012) Paraspinal textiloma after posterior lumbar surgery: a wolf in sheep's clothing. World Neurosurg 77: 375-380.

5. Yamaguchi M, Kumada K (1995) Aseptic encapsulation of retained surgical sponge. Can J Surg 38: 100.

6. Marquardt G, Rettig J, Lang J, Seifert V (2001) Retained surgical sponges, a denied neurosurgical reality? Cautionary note. Neurosurg Rev 24: 41-43.

7. Ramirez LF, Thisted R (1989) Complications and demographic characteristics of patients undergoing lumbar discectomy in community hospitals. Neurosurgery 25: 226-230.

8. Eljebbouri B, Salami M, Gazzaz M, El Mostarchid B (2014) A 23-year-old spinal textiloma after discectomy for lumbar disc herniation. Spine J 14: 2782 .

9. Konya D, Özgen S, Pamir MN (2004) Foreign body mimiking spinal tumor; A case report. Tu“rk Nörosiru“rji Dergisi 3: 191-195. 